\title{
Article
}

\section{Medication adherence early after stroke: using the Perceptions and Practicalities framework to explore stroke survivors', informal carers' and nurses' experiences of barriers and solutions}

Gibson, Josephine, Coupe, Jacqueline and Watkins, Caroline Leigh Available at http://clok.uclan.ac.uk/36492/

Gibson, Josephine ORCID: 0000-0002-3051-1237, Coupe, Jacqueline ORCID: 0000-0002-2025-5620 and Watkins, Caroline Leigh ORCID: 0000-0002-94033772 (2021) Medication adherence early after stroke: using the Perceptions and Practicalities framework to explore stroke survivors', informal carers' and nurses' experiences of barriers and solutions. Journal of Research in Nursing, 26 (6). pp. 499-514. ISSN 1744-9871

It is advisable to refer to the publisher's version if you intend to cite from the work. http://dx.doi.org/10.1177/1744987121993505

For more information about UCLan's research in this area go to http://www.uclan.ac.uk/researchgroups/ and search for <name of research Group>.

For information about Research generally at UCLan please go to http://www.uclan.ac.uk/research/

All outputs in CLoK are protected by Intellectual Property Rights law, including Copyright law. Copyright, IPR and Moral Rights for the works on this site are retained by the individual authors and/or other copyright owners. Terms and conditions for use of this material are defined in the policies page. 


\title{
Medication adherence early after stroke: using the Perceptions and Practicalities Framework to explore stroke survivors', informal carers' and nurses' experiences of barriers and solutions
}

\section{Josephine Gibson (1)}

Reader in Health Services Research, School of Nursing, University of Central Lancashire, Preston, UK

\section{Jacqueline Coupe}

Research Associate, Faculty of Health and Wellbeing, University of Central Lancashire, Preston, UK

\section{Caroline Watkins}

Professor of Stroke and Older People's Care; Faculty Director of Research and Innovation, School of Nursing, University of Central Lancashire, Preston, UK

\begin{abstract}
Background: Secondary prevention medication after stroke reduces risk of recurrence, but adherence is often poor. Stroke survivors', carers' and nurses' perspectives of early poststroke medication adherence are unexplored.

Aim: The aim of this study was to explore stroke survivors', carers' and nurses' views and experiences about adhering to medication early after post-stroke hospital discharge.

Methods: Qualitative individual and group interviews, utilising the Perceptions and Practicalities Framework, were employed. Nine people $<2$ months post-stroke, three carers and 15 nurses from one UK stroke unit participated. Interviews were digitally recorded, transcribed and thematically analysed.

Results: There were four main themes with two sub-themes. (I) Perceptions of medication taking after stroke. Factors affecting adherence included depression, imperceptible benefits and concerns about adverse effects. (2) Perceptions about those at higher risk of poor medication adherence. Nurses suggested that poor adherence might be more likely in those living alone or with previous non-adherence. (3) Practicalities of taking medication early after stroke; these
\end{abstract}


included post-stroke disabilities, cognition, polypharmacy and lack of information. (4a) Practicalities of addressing poor medication adherence during the hospital stay. Solutions included multidisciplinary co-ordination, but nurses and stroke survivors described suboptimal use of opportunities to promote adherence. (4b) Practicalities of addressing poor medication adherence post-discharge. Solutions included modifications and support from carers, but stroke survivors reported difficulties in evolving systems for taking medications.

Conclusions: Stroke survivors and informal carers lack knowledge and support needed to manage medication early after discharge. Nurses' opportunities to promote medication adherence are under-exploited. Medication adherence strategies to support stroke survivors early after discharge are needed.

\section{Keywords}

caregivers, medication adherence, patient discharge, polypharmacy, secondary prevention, stroke

\section{Introduction}

Recurrence after a first stroke is a common, yet often preventable, event. Although there is much heterogeneity in reporting, the 10-year cumulative risk of stroke recurrence in those who survive the initial event has been estimated at 39.2\% (95\% CI, 27.2-51.2) (Mohan et al., 2011). More than a quarter of these recurrent events occur within 12 months, with a cumulative risk of stroke recurrence of $11.1 \%(95 \% \mathrm{CI}, 9.0-13.3)$ at 1 year post-stroke (Mohan et al., 2011), but medication substantially reduces this risk (Hackam and Spence, 2011). Current clinical guidelines recommend several secondary prevention medications which should be started immediately and continued indefinitely (Intercollegiate Stroke Working Party, 2016), but medication adherence is often poorly managed during stroke discharge planning (Andrew et al., 2017).

Poor medication adherence is a global problem which contributes to poor health outcomes across long-term conditions (Yusuf, 2002); only half of prescribed medications are actually taken (Sabate, 2003). Several factors are known to predict poor medication adherence after stroke, including lack of support with medications, polypharmacy, and severe stroke (Al AlShaikh et al., 2016a); anger (Santos and Ferro, 2017) as measured using the State-Trait Anger Expression Inventory-2 (Spielberger, 1999); concerns about treatment (Al AlShaikh et al., 2016a; Crayton et al., 2017); and knowledge and beliefs about medication (Crayton et al., 2017). The contributions of informal carers are also increasingly recognised, but there is a lack of evidence on factors affecting medication adherence in people who rely on such support (De Simoni, Mant and Sutton, 2015).

The development of effective interventions to improve medication adherence is important (Nunes et al., 2009) but challenging (Nieuwlaat et al., 2014), and many studies in stroke are not based on a comprehensive understanding of stroke survivors' needs (Bridgwood et al., 2018; Al AlShaikh et al., 2016b). In order to ascertain the barriers which may affect medication adherence early after stroke, and to develop interventions to overcome them, it is important to understand the perceptions of stroke survivors, informal carers and health professionals. A recent meta-synthesis of 12 qualitative studies, has been conducted on 
stroke survivors', carers' and health professionals' perspectives of medication taking after stroke (Gibson et al., 2020). Of the 412 participants across the included studies, most were stroke/TIA survivors with only $8 \%$ being health professionals.

The meta-synthesis identified a substantial burden of 'medicines work'. This work consists of information work, health care work, carer work, emotional work and practical work, the performance of which necessitates mutual trust, and trust in the benefits of medicines themselves, to overcome barriers to medication adherence and persistence. However, none of the studies identified in this meta-synthesis focused on the period immediately after hospital discharge; instead, they mainly examined long-term medication adherence. Many of the studies in this review also had significant methodological weaknesses, with nine being of moderate and three of poor quality. In particular, few studies have utilised any specific theoretical framework to guide either data collection or analysis. An exception is Jamison et al. (2017) who utilised the Perceptions and Practicalities (PaPa) Framework (Harding and Horne, 2001). This Framework enables exploration of perceptual factors or beliefs about medicines, which may contribute to intentional non-adherence, and practical or resource factors which contribute to non-intentional non-adherence. The PaPa Framework is a particularly suitable model to underpin studies of medication adherence as it has the express purpose of supporting identification and classification of factors influencing medication adherence, with the potential to utilise the findings to guide the development of interventions to address non-adherence. Other models of behaviour change, for example the Theoretical Domains Framework (Cane et al., 2012) could also be useful but are not specifically designed to address the topic of medication adherence.

Medication adherence support is particularly important early after stroke because the risk of recurrent stroke is highest at this time (Mohan et al., 2011), and because early noncommencement of or non-adherence to medications is associated with poor long-term adherence (Thrift et al., 2014; Tsai et al., 2014), yet there are no qualitative studies in the literature that focus on this time period, with people often participating many months or years post-stroke (Gibson et al., 2020). The development of self-management strategies is a priority area for stroke nursing research (Rowat et al., 2016), and could include supported self-management in relation to medication adherence. A case study of one such intervention has suggested that strategies should include targeted education, regular review, and consideration of personal circumstances such as affordability (Kim at al., 2015). The importance of interprofessional practice and provision of support for self-management from nurses and other health professionals has also been identified more generally, in relation to medication adherence for people with chronic disease in primary care (Kvarnström et al., 2018). However, only one previous study (Bauler et al., 2014) has considered the perspectives of nurses, yet their expertise and day-to-day practice in medication management and administration may be valuable in identifying and addressing potential problems.

\section{Aim}

The aim of this study was to utilise the Perceptions and Practicalities Framework (Harding and Horne, 2001) to explore the views and experiences of stroke survivors, informal carers and stroke-unit nurses about challenges and solutions in adhering to medication early after hospital discharge following a stroke. 


\section{Methodology}

\section{Study design}

A qualitative approach was used with semi-structured individual and group interviews. A thematic approach was utilised for data collection and analysis, based on the 'Perceptions and Practicalities' (PaPa) Framework (Harding and Horne, 2001). Major themes were delineated in the interview schedule; sub-themes relating to participants' lived experience were newly identified as the study progressed.

\section{Setting}

The setting was a single stroke unit in England, UK, with acute and rehabilitation facilities at two sites within the same NHS hospital trust, from June 2013 to May 2014.

\section{Sampling and recruitment}

A purposive, maximum variation sample was recruited of stroke survivors, informal carers and registered nurses. Eligibility criteria are shown in Table 1. Nurses with a range of seniority and length of experience were recruited via email and personal contact with the participating stroke unit. We endeavoured to include stroke survivors after first or recurrent strokes, and those who lived alone or with a family member. Before discharge, stroke survivors were given information about the study by a research nurse and were asked for permission to forward their details to the researchers. A researcher telephoned the stroke survivor approximately one week after discharge to arrange a face-to-face interview, to take place within 60 days ( 2 months) of discharge. Informal carers were recruited by asking stroke survivors to give an information leaflet to someone they wished to be present at the interview.

Table I. Eligibility criteria for participation.

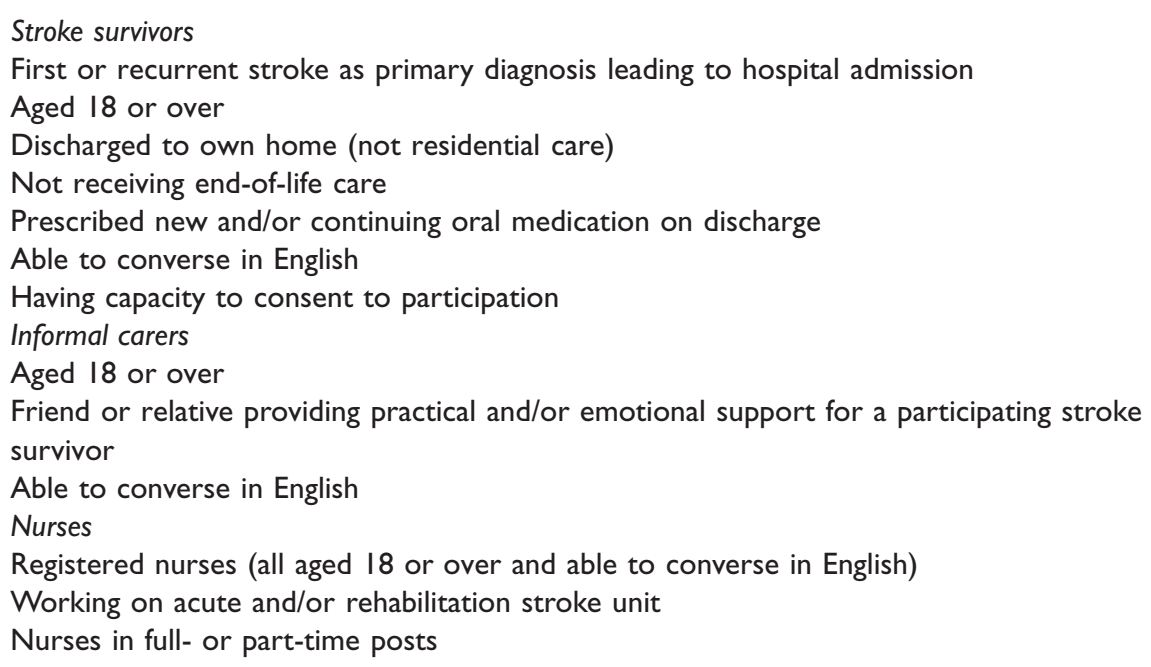


All participants had capacity to consent to participate and all agreed to the use of anonymised direct quotes in reporting of the study findings.

Data collection continued until no new themes were identified. The research team comprised researchers with clinical nursing experience and expertise in stroke research, including qualitative methods. None were working in a clinical role at the time of the study. The lead researcher ( $\mathrm{J}$ Gibson) also holds a post-registration qualification in prescribing.

\section{Data collection}

Semi-structured interviews were conducted by either J Gibson or J Coupe. Interviews with stroke survivors and informal carers were held at stroke survivors' homes. Individual $(n=7)$ or small-group interviews (one interview with two participants; two with three participants each) with nurses were held in a quiet room in the stroke unit. The topic guides, based on the $\mathrm{PaPa}$ framework (Harding and Horne, 2001) included: people's perceptions of factors affecting medication adherence and beliefs about medications after stroke; and their lived experience of the practicalities of taking medication after stroke and of solutions and strategies to address or prevent difficulties. Brief demographic and medical data were also requested from stroke survivors, and data about professional education and experience from nurses. Overall interview duration ranged from 13 to 50 minutes. Data collection took place between June 2013 and May 2014.

\section{Data analysis}

All interviews were digitally audio-recorded and fully transcribed. Initial thematic coding was undertaken using NVivo 10 software. The PaPa framework was used as an overarching structure. Initial codes were clustered via a process of constant comparison, which was undertaken iteratively by $\mathbf{J}$ Gibson and $\mathbf{J}$ Coupe. Coding was compared and discussed where needed to reach agreement. Interpretation of interview content was verified by sharing coding with participants.

\section{Results}

\section{Participants' characteristics}

The 27 participants included 15 nurses (of 40 eligible), all of whom were qualified to diploma $(n=12)$ or bachelor's degree $(n=3)$ level. Most $(10 / 15)$ were staff nurses (NHS Band 5); five were more senior. Ten worked on the acute stroke setting, four in rehabilitation and one across both settings. They typically had 6-10 years' postregistration experience and at least one year's experience in stroke nursing. Nine stroke survivors participated (of 16 approached), of whom six were female and three were male. Their ages ranged from 52 to 76 years (mean 61.8 years). Three informal carers also participated at the invitation of stroke survivors. Characteristics of stroke survivors and carers are detailed in Table 2. Where verbatim quotes are provided, these have been edited where necessary to remove any potentially identifying details, and are identified only by participant number.

Four major themes were identified, the fourth theme having two sub-themes. These and the codes associated with them are summarised in Figure 1. 


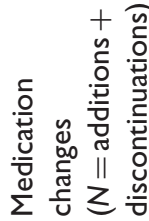

$\operatorname{nnnt.40-n}$

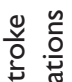

莒

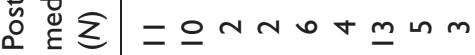

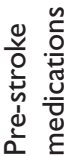

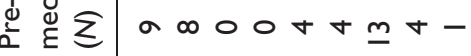

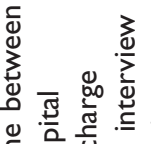

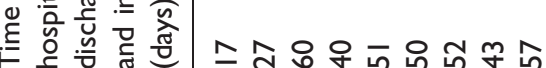

돈

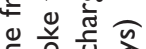

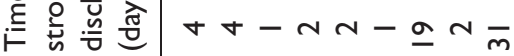

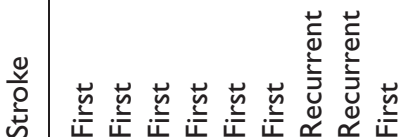

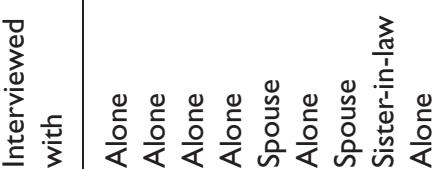

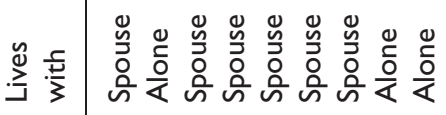

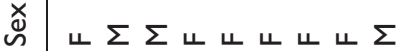

离 ทำกำกำกำก

产

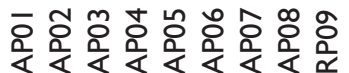




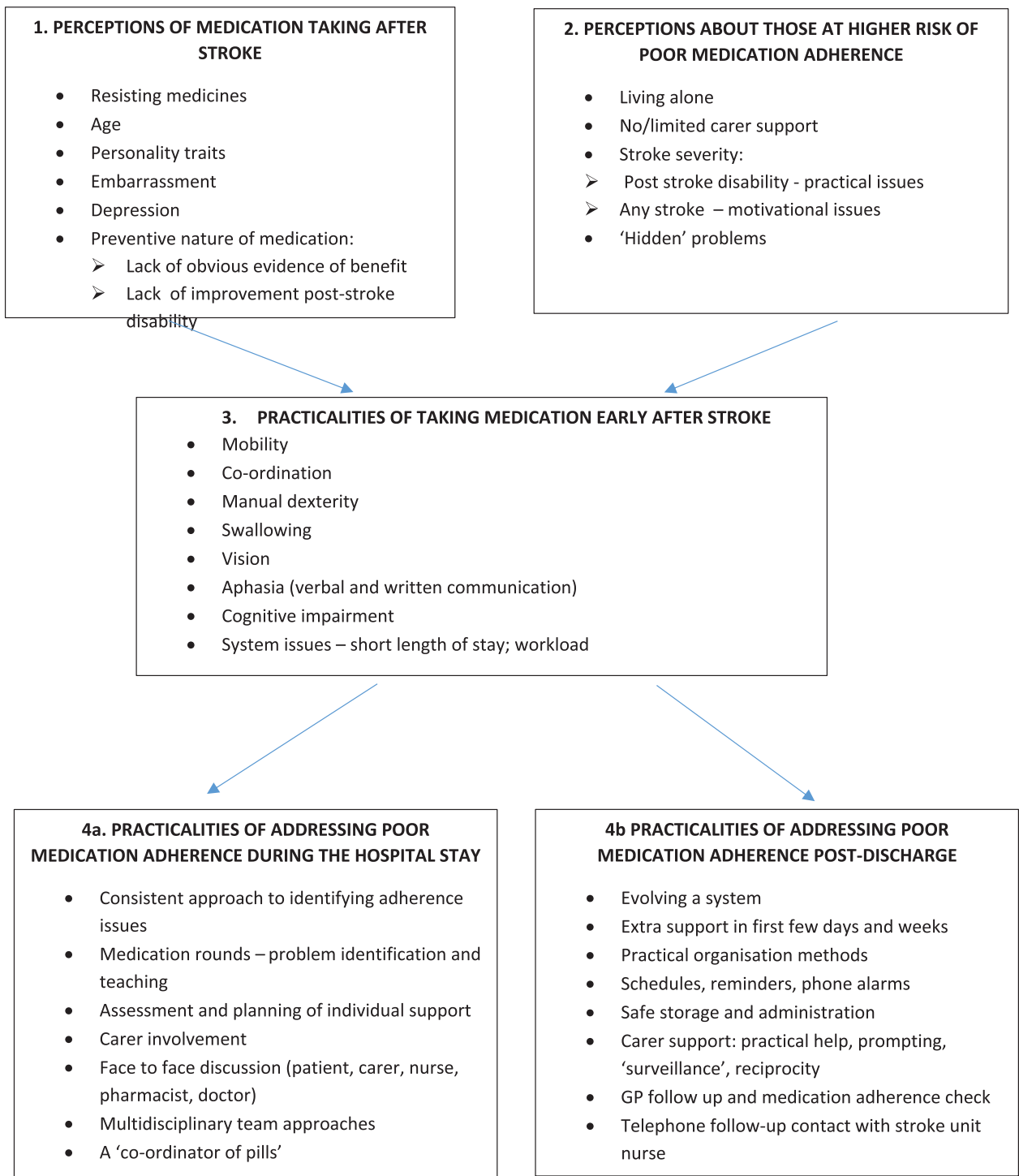

Figure I. Themes identified in interviews with nurses, stroke survivors and informal carers about medication adherence early after stroke.

\section{Theme I: Perceptions of medication taking after stroke}

Codes included in this theme were: resisting medicines; age; personality traits; embarrassment; depression; and the preventive nature of medication - lack of obvious evidence of benefit and lack of improvement post-stroke.

Nurses described a number of barriers relating to stroke survivors' beliefs and concerns about medication. The impact of age on motivation was thought to be variable: whilst some 
older stroke survivors accepted the necessity for medication, others' motivation might be affected by low mood or depression:

'Their whole life's changed and they're really down in the dumps and they don't want to live at all ... (so they think) "What's the point in taking tablets?" ...' (RSO4) (Nurse, rehabilitation unit).

Nurses also felt that younger people might resist the idea of taking medication because of stigma, especially if it was the only obvious reminder of their stroke:

'... if they're ... with family and friends, they'll just feel "Oh I'll have to get all these tablets out of my handbag"...' (ASO2, 03, 04) (Nurses, acute unit).

Resistance to medication might also be related to personality traits:

'... some people can be quite stubborn ... they'll say "Well ... I'm not taking it ... and that's it"” (AS02,03,04) (Nurses, acute unit).

Nurses felt that the lack of obvious benefit with secondary prevention medications contributed to poor adherence and unsupervised discontinuation. They noted that motivation and adherence might be higher with warfarin than with other drugs because the necessary regular monitoring of coagulation provided ongoing evidence of its effectiveness. For stroke survivors, evidence that medication was 'vitally important' was inherent in its implicit endorsement by health professionals and the health service, '.. otherwise why would you be given it?' ( RP09).

Generally, stroke survivors believed that it was important to take medication, but when asked if they felt that the medication was beneficial, many of them hesitated before answering. Absence of a further stroke and of significant side effects were seen as indicators of benefit:

'I feel okay ... the medication is doing what it is supposed to be doing ... I know that it thins the blood and ... it hopefully will prevent having another [stroke]' (AP04).

One survivor of a recurrent stroke, who was prescribed 13 different medications, felt that this polypharmacy was a negation of her efforts to rehabilitate after her first stroke:

'I shouldn't have to take all them, you want to feel like you're doing well in life, don't you, but you're not well ... I fought through the first stroke, for my speech and movement and walking, and then I've had another [stroke] and I've been fighting through that ... and then I'm taking more pills' (AP07, ACO2).

Similarly, nurses identified that stroke survivors might feel unmotivated to continue medications if there was little sign of physical recovery:

'You have, every day, ten tablets... (but) ... you don't feel any better ... your arm is still without movement ... and [you think] "I'm taking tablets every day ... there is no point in taking it"." (ASO8, 09, 10) (Nurses, acute unit).

\section{Theme 2: Perceptions about those at higher risk of poor medication adherence}

Codes included in this theme were: living alone; no/limited carer support; stroke severity post-stroke disability/practical issues; motivational issues; and 'hidden' problems. This theme included both social and clinical characteristics. Nurses were aware of many 
possible predictors of adherence problems, but their identification of these potential problems in clinical practice was unsystematised and sporadic.

Nurses felt that living alone was a risk factor for poor adherence, and, indeed, all stroke survivors in the study, irrespective of their living arrangements, received informal support to obtain, organise or remember to take medication. This practical assistance was also a tangible expression of the informal carer's emotional support and concern:

'... I'll tell him [husband] - you don't need to remind me, I know what I'm doing ... but I think it's a shock for them as well ... just for that first ... two or three weeks ... he's probably thinking it's better to remind me to make sure...' (AP04).

Nurses felt that stroke severity impacted on adherence in various ways. Physical or cognitive problems might directly cause practical difficulties and could also lead to incorrect assumptions about the futility of preventive measures. Conversely, in people who had recovered fully, denial and lack of understanding of the potential seriousness of the stroke might also lead to poor adherence.

Nurses felt that past problems with medication adherence, or numerous queries from a stroke survivor about their medication while in hospital, suggested a lack of understanding or confidence and might predict poor post-discharge adherence. Nurses would also suspect poor prior adherence in stroke survivors who expressed reluctance to take medication, or where there was direct evidence:

'Sometimes they come in with tablets that are out of date, you know they've had them years ...' ( ASO2, 03, 04) (Nurses, acute unit).

Although stroke survivors and informal carers were not directly asked about how people's risk of poor medication adherence might be identified, some who initially stated that they had not had any adherence problems then acknowledged such difficulties later in the interview. This suggests that current and potential medication adherence problems might easily be overlooked by health professionals:

'It's not been a problem really at all' (AP01) - later described forgetting to take medication, difficulties with splitting tablets into quarters and with swallowing.

'No problems with them ... none at all, and I don't think there's any side effects' (RP09) - later described difficulties with manual dexterity, opening packets and dropping tablets.

\section{Theme 3: Practicalities of taking medication early after stroke}

Codes included in this theme were: mobility; co-ordination; manual dexterity; swallowing; vision; aphasia (verbal and written communication); cognitive impairment; and system issues (short length of stay; workload).

People with limited mobility experienced difficulties in obtaining their prescriptions and supplies of medication, and also had to identify a secure but accessible place to store medications at home. Post-stroke problems with co-ordination and dexterity were also a barrier:

'... the (medication) packets are a pain in the neck ... I'm one-handed initially, so I didn't use my left hand' ( RP09).

'If ... nobody thought to put them (medications) within reach, then he wouldn't be able to get them' ( RSO2) (Nurse, rehabilitation unit). 
Post-stroke dysphagia was mentioned by all nurses as a potential barrier. None of the stroke survivors had persistent dysphagia, but those who had transient problems early after their stroke still had a fear of tablets getting 'stuck'.

Some stroke survivors reported difficulties in reading medication labels, and nurses recognised that visual impairments were a potential barrier:

‘... you've got to make sure they can read (the labels) ... before they get home ... you could have a hemianopia' (AS11) (Nurse, acute unit).

Nurses suggested that aphasia could be a barrier to medication adherence, making it challenging for health professionals or other carers to assess understanding, and for stroke survivors to communicate difficulties or queries:

'... they might say no [to medication] and they mean yes...' (RSO2) (Nurse, rehabilitation unit).

Other barriers suggested by nurses included post-stroke cognitive impairment, especially when combined with polypharmacy; likewise, stroke survivors, none of whom had significant cognitive impairment, also described difficulties with remembering to take medication, or even omitting a new medication altogether:

'There was a great big bag with all the boxes in ... and one of the boxes we missed ... I don't remember them saying you're on two lots of tablets...' ( AP01).

Organisational barriers to adherence included poor communication at admission and discharge, short lengths of hospital stay, and lack of availability of nurses to discuss medications. Pressure on bed availability could lead to rushed discharges and communication failures:

'We're supposed to sit with the patient and go through the discharge summary ... (but) new admissions are here even before the old patient has left the ward' (AS07) (Nurse, acute unit).

Similarly, stroke survivors' reports of information provision were variable:

‘... on the day I was going [home], she [nurse] gave me full details about the medication ... we had a question and answer session about my understanding ...' (AP03).

"[nurse] said "take these" ... it could have been anything ... perhaps they could have told me ... what they [tablets] were actually going to do' (AP01).

\section{Theme 4}

This theme addressed the practicalities of addressing medication adherence after stroke, in the light of insights from themes 1-3. During analysis, it became clear that the overall theme fell into two areas: (a) pre-emptive measures that could be implemented during the hospital stay, and (b) measures that could be used after discharge to address emergent and ongoing medication adherence issues. We thus created two subthemes.

Theme 4a: Practicalities of addressing poor medication adherence during the hospital stay. Codes included in this theme included: a consistent approach to identifying adherence issues; medication rounds for problem identification and teaching; assessment and planning of 
individual support, carer involvement; face-to-face discussion (patient, carer, nurse, pharmacist, doctor); multidisciplinary team approaches; and having a 'co-ordinator of pills'.

Solutions suggested by nurses to improve stroke survivors' medication adherence included incorporating education in medication rounds, regime simplification, individual assessment and planning, carer involvement, and multidisciplinary communication:

'We could do with a multidisciplinary team meeting with a nurse, patient and a relative and the pharmacist ... it has to be on an individual basis 'cos everyone's different' (AS05, 06) (Nurses, acute unit).

In practice, however, pre-discharge support was uncoordinated and often rushed or even omitted due to time pressures:

'Every patient that's discharged we go through them [the medication] - well, I do, I assume everybody else does!' (AS01) (Nurse, acute unit).

'... we go through it [discharge summary] with the main carer ... but we don't always have time to go through everything properly' (RSO1) (Nurse, rehabilitation unit).

Likewise, a stroke survivor highlighted the need for a 'co-ordinator of pills':

'just invent one person that can put it all together ... I just wish there was somebody ... what do you call 'em, co-ordinator of pills [laugh]' ( AP07, AC02).

Theme 4b: Practicalities of addressing medication adherence issues after discharge. Codes included in this theme were: evolving a system; extra support in the first few days and weeks, practical organisation methods; schedules, reminders, phone alarms; safe storage and administration; carer support (practical help, prompting, 'surveillance', reciprocity); general practitioners (GP) follow up and medication adherence checks; and telephone follow-up contact with stroke-unit nurse.

Stroke survivors and informal carers developed diverse solutions and adaptations to address medication adherence difficulties after returning home, including purpose-made or improvised pill organisers, schedules, electronic, written or personal reminders, and strategies to ensure safe administration and storage:

'I'm one-handed, trying to get it out and not have the tablet go at ninety miles an hour ... across the floor, and I'm worrying about my dog ... (I have to make sure) the dog's not in the room ... I dropped it once and found it straight away...'( RPO9).

Informal carers often provided active prompting. This was seen as 'automatic' (AP06) but was not always wholly welcomed:

'At the beginning he said "have you taken (your medication)?". .. just checking up ... and he won't stop doing it ... he goes over the top a bit' ( AP04).

Provision of weekly prepacked blister packs also facilitated discreet surveillance and support from the carer:

'I just presumed she were getting on with it and she kept forgetting them ... now I can glance down (at the blister pack) ... and know that she's took the morning ones ... it just makes my life easier' ( AP07, AC02). 
In some cases, this support was reciprocated by the stroke survivor:

'I [carer] take medication, and I forget mine if I'm not careful and [name] reminds me, so we look after each other' (AP05, AC01).

'(after dinner my husband) says "What are you taking?" and I'll say "I'm taking a statin," [laughs] and he says 'I'm taking my statin now too ... is this a pudding?" [laughs]' (AP04).

Informal carers also provided practical support to obtain repeat prescriptions or to attend monitoring appointments:

'It's not been a hassle for me (blood tests) because ... my sister-in-law took me to hospital ... because you just can't walk that [far]' (AP08, AC03).

Nurses suggested that telephone support after discharge, initiated by either party, might be helpful:

'In a couple of weeks, just ring the patient up and say ... "any problems with your medicines, are you taking them?"' (AS05, 06) (Nurses, acute unit).

'Give them a telephone number if they're worried (about medications), tell them - "Please contact us, we're here twenty-four hours (a day)'”' (RSO4) (Nurse, rehabilitation unit).

GPs were also cited as a potential source of post-discharge medication support, but several participants reported poor communication, exacerbated by time pressures:

'The doctor sent me a letter for a review of my medication ... and all she did were put it up on the computer, looked at it and said "Right, we don't have to change anything," ... I really think they're missing a few things because ... they've only twenty minutes to see a patient' (AP07, AC02).

\section{Discussion}

This study is the first to highlight the complex challenges faced by stroke survivors and informal carers in evolving a system to manage medication early after discharge. It is also the first study to focus on the contributions of stroke-unit nurses to addressing these challenges. Stroke-unit nurses', stroke survivors' and carers' perceptions of medication adherence echo the issues of lack of support, polypharmacy (Al AlShaikh, 2016a), and the need for trust in medicines (Gibson et al., 2020), and this trust is also implicit in stroke survivors' acceptance of the endorsement of medicines by health professionals.

Nurses', stroke survivors' and carers' views were mostly reflective of each other, notably in relation to the lack of obvious benefit of secondary prevention, the impact of physical stroke sequelae such as manual dexterity and dysphagia, the importance of carer support, and organisational factors. Delivery of education and support by nurses about post-stroke medication was recognised as important, but both nurses' and stroke survivors' experiences of this were variable.

Our study highlights that the challenges faced by stroke survivors on returning home stem from the need for rapid transition, from being passive recipients of medication, to selfmanagement of often complex medication regimes, and the multiple activities relating to this medicines work. These include initiating medication reviews, organising and undergoing necessary monitoring, organising and obtaining repeat prescriptions, storing medications safely, taking medications on schedule and resolving any problems. Far from the ideal of supported self-management (Rowat et al., 2016), this rapid, largely unsupported transition 
adds significantly to the treatment burden (Gallacher et al., 2019) shouldered by stroke survivors and informal carers in the first weeks after discharge, when the underlying risk of recurrent stroke is highest and the need to establish a consistent and manageable medication regime is greatest. In this study, nurses also appeared to recognise many of the potential problems that stroke survivors also identified and that have been reported in other studies (Al AlShaikh et al., 2016a), such as polypharmacy and the lack of support, and described how they attempted to resolve them via opportunistic assessment and individualised support. However, if this area of nursing knowledge and practice is not recognised formally in the work of the stroke unit, nurses are unable to provide the comprehensive advice and support needed.

This qualitative study adds to our understanding of the challenges faced by stroke survivors, carers and nurses around medication adherence early after stroke, and suggests some possible solutions which are worthy of further investigation. By using the $\mathrm{PaPa}$ framework (Harding and Horne, 2001) the findings capture practical problems and complex strategies, and portray how people develop solutions by trial and error in the early days at home after a stroke. Although previous qualitative studies (Bauler et al., 2014; Souter et al., 2014; Jamison et al., 2016) have highlighted the contributions of various professional groups in post-stroke medication adherence, this study is the first to focus on the contribution of nurses working in inpatient stroke settings, synthesising their perspectives with those of stroke survivors and informal carers to formulate a shared understanding which may contribute to the development of collaborative, multidisciplinary solutions.

The study has a number of limitations. It was a small single-centre study conducted with stroke survivors, informal carers and nurses from only one stroke unit. Thus, although we were able to develop the key themes of the $\mathrm{PaPa}$ framework in some depth in relation to post-stroke medication taking, exploration of other professionals' experiences and views, notably those of community nurses and other members of the stroke multidisciplinary team, might have led to the development of further sub-themes. Caution should be exercised in generalising the findings to other settings and in considering transferability of the recommendations. Notably, no participants mentioned the cost of medicines, perhaps because prescription medications in the UK National Health Service are provided free or for a small flat-rate payment, but in some other health care systems, medication costs may have more impact on adherence (Lummis et al., 2008). We found during recruitment that stroke survivors who did not acknowledge having difficulties with their medication were perhaps less interested in participating, so the study may over-represent the views of those who did experience problems. The problems disclosed by stroke survivors related almost entirely to unintentional non-adherence, and few inferences can be made about intentional non-adherence. Although the study protocol allowed for the recruitment of people with cognitive or communication disabilities after stroke in light of the potential impact of these on adherence, we were not successful in recruiting any such participants. Only a small number of informal carers agreed to participate, and none of them supported a stroke survivor who was wholly reliant on them for medication taking. Exploration of the perspectives of community nurses who support stroke survivors would have also been valuable to enable an understanding of how nurses contribute to post-stroke medication management on both sides of the discharge process. This is particularly pertinent given the recent development of 'early supported discharge' approaches, in which nurses play key roles in dedicated multidisciplinary community stroke teams. 
Although the findings of this small, single-centre study should be interpreted with caution, they suggest several areas for further research. Multidisciplinary team approaches to poststroke medication adherence need to be developed and tested, spanning both inpatient and community settings. These should focus on education, training and support for both stroke survivors and carers where appropriate. The views and experiences of carers, especially those who support a person with severe post-stroke disability, and of nurses working in community settings, are also under-researched.

\section{Conclusion}

This study has highlighted stroke survivors', carers' and stroke nurses' perceptions of taking medication early after discharge, perceptions about those at risk of poor medication adherence, the practicalities of managing medication at this time, and the practicalities of identifying and addressing potential and emerging problems. Perceptions of post-stroke medication are influenced by the lack of obvious benefit derived from secondary prevention medication, in the context of ongoing stroke disability and other factors such as age and embarrassment. Those living alone, with limited support, and with specific poststroke disabilities, including hidden disabilities, or with low motivation, are perceived to be most at risk. After discharge, stroke survivors and carers have to address the practicalities of managing medications, often with little or no preparation. Practical methods to proactively address poor medication adherence during the hospital stay need to use a consistent, multidisciplinary and co-ordinated approach, with comprehensive individualised assessment and support. Practical methods utilised by stroke survivors and carers after discharge centre on 'evolving a system', including scheduling, safe storage and carer support. Follow-up support by the stroke unit and from primary care are also important but inconsistently delivered.

Stroke survivors are at risk of avoidable medication adherence problems early after discharge. This reduces the efficacy and safety of their prescribed medications and contributes to stroke recurrence in both the short and longer term. Systematic multidisciplinary systems of support, within which nurses contribute to identifying potential problems and supporting medication adherence, might facilitate adherence early after discharge. The contributions of informal carers may be vital to ensuring medication adherence after stroke and should also be integrated into the stroke survivor's care plan where appropriate.

\section{Key points for policy, practice and/or research}

- Numerous practical and motivational problems affect medication adherence early after stroke.

- Stroke survivors have to evolve a system largely unaided to manage their medications effectively.

- The expertise of stroke-unit nurses in this area is not well-used.

- Multidisciplinary strategies to support stroke survivors with medication adherence early after discharge need to be developed and evaluated. 


\section{Declaration of conflicting interests}

The author(s) declared no potential conflicts of interest with respect to the research, authorship, and/or publication of this article.

\section{Ethics}

The study received ethical approval from the host academic institution's ethical review committee, the National Research Ethics Service (reference 12/NW/0728), and the hospital's Research and Development Department. Written informed consent was obtained from all participants. Participant data was anonymised and securely held in electronic form on a secure password-protected server at the host academic institution. The COREQ guidelines were consulted in reporting of the study.

\section{Funding}

The author(s) disclosed receipt of the following financial support for the research, authorship, and/or publication of this article: This work was supported by the General Nursing Council Trust for England and Wales (grant number). CW is partly funded by the National Institute for Health Research Applied Research Collaboration North West Coast. The views expressed are those of the authors and not necessarily those of the NIHR, NHS or Department of Health and Social Care.

\section{ORCID iD}

Josephine Gibson (D) https://orcid.org/0000-0002-3051-1237

\section{References}

Al AlShaikh S, Quinn T, Dunn W, et al. (2016a) Predictive factors of non-adherence to secondary preventative medication after stroke or transient ischaemic attack: A systematic review and meta-analyses. European Stroke Journal 1(2): 65-75.

Al AlShaikh S, Quinn T, Dunn W, et al. (2016b) Multimodal interventions to enhance adherence to secondary preventive medication after stroke: A systematic review and metaanalyses. Cardiovascular Therapeutics 34(2): 85-93.

Andrew NE, Busingye D, Lannin NA, et al. (2017) The quality of discharge care planning in acute stroke care: Influencing factors and association with postdischarge outcomes. Journal of Stroke and Cerebrovascular Diseases 27(3): 583-590.

Bauler S, Jacquin-Courtois S, Haesebaert J, et al. (2014) Barriers and facilitators for medication adherence in stroke patients: A qualitative study conducted in French neurological rehabilitation units. European Neurology 72(5-6): 262-270.

Bridgwood B, Lager KE, Mistri AK, et al. (2018) Interventions for improving modifiable risk factor control in the secondary prevention of stroke. Cochrane Database of Systematic Reviews 5(5): CD009103, DOI: 10.1002/ 14651858.CD009103.pub3.

Cane J, O'Connor D and Michie S (2012) Validation of the theoretical domains framework for use in behaviour change and implementation research. Implementation Science 7: 37, DOI: $10.1186 / 1748-5908-7-37$.

Crayton E, Fahey M, Ashworth M, et al. (2017) Psychological determinants of medication adherence in stroke survivors: A systematic review of observational studies. Annals of Behavioral Medicine 51(6): 833-845.

De Simoni A, Mant J and Sutton S (2015) Adherence to medication in stroke survivors dependent on caregivers. British Journal of General Practice 65(640): e785-e789.

Gallacher K, Quinn T, Kidd L, et al. (2019) Systematic review of patient-reported measures of treatment burden in stroke. BMJ Open 9(9): e 029258, DOI: 10.1136/bmjopen-2019029258.

Gibson JME, Miller C, Coupe J, et al. (2020) Medicationtaking after stroke: A qualitative meta-synthesis of the perspectives of stroke survivors, informal carers, and health professionals. Family Practice 37(1): 4-14.

Hackam DG and Spence JD (2007) Combining multiple approaches for the secondary prevention of vascular events after stroke: A quantitative modeling study. Stroke 38(6): 1881-1885.

Harding KTG and Horne R (2001) Compliance, adherence and concordance. In: Harding KTG (ed.) Pharmacy Practice. London: Taylor \& Francis, pp. 148-169.

Intercollegiate Stroke Working Party (2016) National Clinical Guideline for Stroke, 5th ed. London: Royal College of Physicians.

Jamison J, Graffy J, Mullis R, et al. (2016) Barriers to medication adherence for the secondary prevention of stroke: A qualitative interview study in primary care. British Journal of General Practice e568, DOI: 10.3399/bjgp16X685609.

Jamison J, Sutton S, Mant J, et al. (2017) Barriers and facilitators to adherence to secondary stroke prevention medications after stroke: Analysis of survivors and 
caregivers views from an online stroke forum. BMJ Open 7(7): e016814, DOI: 10.1136/bmjopen-2017-016814.

Kim J, Thrift AG, Nelson MR, et al. (2015) Personalized medicine and stroke prevention: Where are we? Vascular Health and Risk Management 11: 601-611.

Kvarnström K, Airaksinen M and Liira H (2018) Barriers and facilitators to medication adherence: A qualitative study with general practitioners. BMJ Open 8(1): e015332,

DOI:10.1136/bmjopen-2016-015332.

Lummis HL, Sketris IS, Gubitz GJ, et al. (2008) Medication persistence rates and factors associated with persistence in patients following stroke: A cohort study. BMC Neurology 8: 25, DOI: $10.1186 / 1471-2377-8-25$.

Mohan KM, Wolfe CDA, Rudd AG, et al. (2011) Risk and cumulative risk of stroke recurrence: A systematic review and meta-analysis. Stroke 42(5): 1489-1494.

Nieuwlaat R, Wilczynski N, Navarro T, et al. (2014)

Interventions for enhancing medication adherence. Cochrane Database of Systematic Reviews 2014(11): CD000011, DOI: 10.1002/14651858.CD000011.pub4.

Nunes V, Neilson J, O'Flynn N, et al. (2009) Clinical Guidelines and Evidence Review for Medicines Adherence: Involving Patients in Decisions about Prescribed Medicines and Supporting Adherence. London: National Collaborating Centre for Primary Care and Royal College of General Practitioners.
Rowat A, Pollock A, St George B, et al. (2016) Top ten research priorities relating to stroke nursing: A rigorous approach to establish a national nurse-led research agenda. Journal of Advanced Nursing 72(11): 2831-2843.

Sabate E (2003) Adherence to Long-Term Therapies: Evidence for Action. Switzerland: World Health Organization.

Santos AC and Ferro JM (2017) The impact of anger in adherence to treatment and beliefs about disease 1 year after stroke. Journal of Neurology 264(9): 1929-1938.

Souter C, Kinnear A, Kinnear M, et al. (2014) Optimisation of secondary prevention of stroke: A qualitative study of stroke patients' beliefs, concerns and difficulties with their medicines. International Journal of Pharmacy Practice 22(6): 424-432.

Spielberger C (1999) Staxi-2: State-Trait Anger Expression Inventory-2: Professional Manual. Lutz, FL: Psychological Assessment Resources Inc.

Thrift AG, Kim J, Douzmanian V, et al. (2014) Discharge is a critical time to influence 10-year use of secondary prevention therapies for stroke. Stroke 45(2): 539-544.

Tsai JP, Rochon PA, Raptis S, et al. (2014) A prescription at discharge improves long-term adherence for secondary stroke prevention. Journal of Stroke and Cerebrovascular Diseases 23(9): 2308-2315.

Yusuf S (2002) Two decades of progress in preventing vascular disease. Lancet 360(9326): 2-3.

Josephine Gibson is a reader in health services research, registered nurse and non-medical prescriber with a particular interest in stroke prevention, on which she has authored reviews and book chapters.

Jacqueline Coupe's research interests focus on the impact of active ageing on the health and well-being of older people. She has also co-authored two Cochrane systematic reviews related to stroke.

Dame Caroline Watkins is a nursing professor of stroke care at the University of Central Lancashire, and was awarded a DBE for Services to Nursing and Older People's Care in 2017. 